Meta

Journal des traducteurs

Translators' Journal

\title{
« Harap's New Standard French and English Dictionary »
}

\section{René Ledésert}

Volume 18, numéro 1-2, mars 1973

Actes du deuxième colloque international de linguistique et de traduction. Montréal, 4-7 octobre 1972

URI : https://id.erudit.org/iderudit/003442ar

DOI : https://doi.org/10.7202/003442ar

Aller au sommaire du numéro

Éditeur(s)

Les Presses de l'Université de Montréal

ISSN

0026-0452 (imprimé)

1492-1421 (numérique)

Découvrir la revue

Citer cet article

Ledésert, R. (1973). « Harap's New Standard French and English Dictionary ». Meta, 18(1-2), 255-260. https://doi.org/10.7202/003442ar d'utilisation que vous pouvez consulter en ligne.

https://apropos.erudit.org/fr/usagers/politique-dutilisation/ 


\section{«Harrap's New Standard French and English Dictionary"}

Je ne suis ni linguiste, ni lexicographe. Les circonstances seules m'ont amené à m'occuper de dictionnaires bilingues. Et, à part ma licence de droit suivie d'une étude du droit anglais pour une thèse de doctorat, que je n'ai jamais terminée à cause de la guerre, je n'avais fait qu'une licence d'allemand. C'est donc avec beaucoup de trépidation que je parle maintenant devant cet aréopage distingué.

Il se trouve que Harrap's New Standard French and English Dictionary, partie français-anglais, a été publié le 2 octobre 1972 en Angleterre et au Canada, en deux volumes de 600 pages chacun A-I et J-Z. Avant de parler de ce nouvel ouvrage, je voudrais, si vous me le permettez, faire l'historique de son prédécesseur, Harrap's Standard French and English Dictionary, qui a paru en un volume de 900 pages en 1934. Et d'abord, parlons de M. Jean Edmond Mansion, son auteur que, malheureusement, je n'ai jamais connu puisqu'il est mort en 1942.

Mansion est né en 1870 à Dreux sur les confins de la Normandie et de l'île-de-France, au moment du siège de Paris par les Prussiens. Son père Horace Mansion était français, et il était un excellent musicien, organiste à l'église principale de Dreux. Sa mère Sarah Douglas était écossaise née à Dalry, Kirkcudbrightshire, et était venue à Dreux comme gouvernante dans une famille noble. Ils se sont rencontrés et se sont mariés. Hélas! Sarah mourut en 1879 , laissant quatre jeunes enfants dont l'aîné était Jean Edmond qui avait à peine neuf ans. Leur père, Horace Mansion ne pouvait pas s'occuper de cette petite famille. Il semble que la sœur de Sarah, Barbara, ait emmené ces jeunes enfants dans sa famille à Dalry et qu'ils y soient restés deux ou trois ans. Puis Horace Mansion épousa une Française et vint chercher ses enfants qui vécurent à Dreux à partir de ce moment-là. Jean Edmond Mansion fréquenta alors le lycée et son éducation fut celle d'un jeune Français de cette époque. Selon sa fille, il semble que la bellemère ait été acariâtre et que Jean Edmond ait réussi à secouer ce joug, envoyant sa sœur Cécile dans une École normale d'où elle sortit comme institutrice.

Jean Edmond continuait ses études à cette époque et il devint bachelier, puis il alla à l'Université de Marburg en Allemagne, mais dût abandonner ses études pour gagner sa vie. Pendant toute cette période il venait passer ses vacances à Dalry. Il devint alors professeur à Dollar Academy, puis il épousa Annie Crosbie en 1901. Vers cette époque il prit la nationalité britannique et c'est alors qu'il changea la prononciation de son nom. 
Le jeune ménage alla ensuite à Belfast, mais nous ne savons pas dans quel collège il enseignait, puis en 1908 ils revinrent en Écosse à Édimbourg où Jean Edmond était le directeur de la section des langues vivantes à George Watson's Academy. Il y resta jusqu'en 1914. Il avait fait sur ces entrefaites la connaissance de M. George Harrap et de M. George Oliver Anderson (cofondateurs de la maison Harrap), et vers 1912 ou 1913 il entreprit le travail du dictionnaire. Son intention à l'origine était de faire mieux et plus complet que tous les dictionnaires anglais-français de l'époque. À Édimbourg, il fit la connaissance d'un jeune licencié d'anglais, Marcel Ferlin, qui travaillait pour son agrégation et qui était assistant dans un collège d'Édimbourg. Et selon M. Ferlin, qui est malheureusement mort en 1970 alors qu'il corrigeait les épreuves du New Standard, l'intention de M. Mansion était bien dès le début d'en faire ce que nous connaissons de l'édition de 1934, c'est-à-dire un dictionnaire trois ou quatre fois plus complet que tous les dictionnaires français-anglais et qui jusqu'à ce jour est resté le plus moderne de tous les dictionnaires.

Selon les personnes qui l'ont connu, il préférait travailler chez lui, le soir ou les samedis et dimanches, loin de la routine du bureau et du téléphone, entouré de sa famille, et écoutant de la musique. Il s'était fait faire un fauteuil dans les bras duquel il pouvait puiser pour trouver les livres de référence dont il avait un besoin constant. Au début on parlait de faire le dictionnaire en trois ans, peut-être cinq. En fait, il fallut vingt et un ans pour que le dictionnaire puisse paraître. Que ceci serve d'avis à tous les lexicographes et éditeurs qui croient qu'un dictionnaire peut se faire en un tour de main! C'est d'ailleurs pourquoi, à part le dictionnaire français-anglais de Mansion, le Harrap's Standard German and English Dictionary (dont deux volumes sur huit ont paru jusqu'à présent), et le Harrap-Sansoni Standard Italian-English Dictionary (dont un volume sur quatre a paru jusqu'à présent), il n'y a pas d'autres dictionnaires bilingues de cette taille ni de cette qualité. En effet l'investissement nécessaire pour ce travail est colossal, et pendant des années on engouffre des millions sans recevoir en compensation le produit des ventes.

Mais revenons à Mansion. Au printemps de 1914 il fut nommé rédacteur en chef des textes scolaires en langues vivantes de la maison Harrap. Puis vint la guerre. Mansion devint professeur à Merchant Taylor's School, mais après sa journée d'enseignant, il se remettait au dictionnaire. J'ai connu le $\mathrm{D}^{\mathrm{r}}$ Nairn qui était le directeur de Merchant Taylor's School à cette époque. Ce monsieur est mort il y a une dizaine d'années, et cette anecdote n'a pas, je crois, été mentionnée nulle part jusqu'à présent. Dans toutes les public schools anglaises, le professeur de français est considéré par les élèves comme une proie facile pour l'organisation d'un chahut monstre. M. Mansion était très petit selon ce que l'on m'a dit, mais aux premières velléités de désordre il fit preuve d'une poigne de fer, et après cela ses élèves lui donnèrent le surnom affectueux de Maisonnette.

Comme je l'ai dit plus haut, M. Mansion est mort en 1942, et jusqu'à la fin il a travaillê, car il avait commencé la rédaction du Harrap's Shorter French and English Dictionary dont la partie français-anglais a paru en 1940. Nous 
avons révisé cet ouvrage depuis — c'est le Harrap's New Shorter French and English Dictionary.

Dans le Mystic Masseur par Naipaul, le célèbre auteur né dans l'île de la Trinité, son héros Ganesh «était devenu un connaisseur en ce qui concernait l'odeur du papier » (il était à ce moment-là en train d'acquérir une bibliothèque avant de se présenter aux élections). Il dit à Beharry (son ami) \& Tu sais je peux sentir un livre et te dire son âge », et il considérait que le livre qui avait la meilleure odeur était Harrap's Standard French and English Dictionary, qu'il avait acheté «simplement pour son odeur». Je suis d'accord que l'odeur du Harrap, tout au moins dans les premières impressions était remarquablement agréable. Mais ce n'est pas la raison pour laquelle je suis venu chez Harrap.

Lorsque j'enseignais les langues vivantes à Eton College, j'avais utilisé constamment le Harrap Standard French and English Dictionary d'une part, et, d'autre part, les livres de langues vivantes publiées par Harrap, qui figuraient pour les trois quarts dans les programmes. Quand en 1945 j'ai songé à changer d'orientation j'ai écrit à Harrap pour demander si par hasard la maison aimerait m'employer pour la préparation des livres scolaires. Deux ou trois jours plus tard j'ai reçu une réponse de George Oliver Anderson, déjà mentionné plus haut, qui était alors le président de la maison, m'invitant à aller le voir. "Monsieur Ledésert, dit-il, nous avons une situation qui vous conviendrait précisément, mais il faut aussi quelqu'un qui pourra réviser le Standard Dictionary. » Puis il me surprit par cette question étonnante : «Êtes-vous philatéliste?» «- Oui.» «- Est-ce que vous vous occupez des filigranes, des perforations, et des variétés?» «- Oui.» «- Dans ce cas je crois que vous pourrez être lexicographe. Voyezvous, la lexicographie c'est un peu comme la philatélie, on remplit des cases vides, on ajoute des exemples, etc. »Et voici, mesdames et messieurs, pourquoi je ne suis pas un lexicographe, mais un philatéliste qui a mal tourné. Hélas ! ma collection de timbres a été complètement négligée depuis cette époque.

C'est donc ainsi que j'ai commencé mon travail chez Harrap, le $\mathbf{1}^{\text {er }}$ janvier 1946, d'abord comme rédacteur en chef, ensuite, depuis 1956, comme administrateur de la maison. Je me suis occupé pendant toutes ces années de la publication des livres destinés à l'enseignement du français, de l'allemand, de l'espagnol et même de l'italien. Mais dès 1947 je me suis mis à la révision du dictionnaire. Le fichier de Mansion avait disparu pendant la guerre au cours d'un raid aérien. Il a d'abord fallu le reconstituer. Nous avons publié le résultat de nos recherches initiales dans plusieurs suppléments. Le premier supplément a paru en 1950, suivi par un second supplément en 1955 et un troisième en 1961. Le volume français-anglais du Standard Dictionary était encore récent en 1946 puisqu'il avait été publié en 1934, mais en raison de la guerre, des nouvelles inventions dans tous les domaines, et du développement des deux langues depuis la langue littéraire, ou celle de tous les jours, ou encore l'argot, nous avons été amenés à augmenter l'ouvrage d'environ 30000 articles (l'édition originale comprenait à peu près 70000 articles) et portant ainsi le total à 100000 articles, et nous avons modernisé et amplifié les articles publiés en 1934. En fait le New Standard 
comprend environ $50 \%$ à $60 \%$ de texte supplémentaire par rapport à l'édition originale.

Pour ce travail nous avons eu recours à bien des sources différentes : tout d'abord les dictionnaires unilingues les plus modernes, car il n'existe pas de dictionnaire bilingue général de cette taille qui ait pu nous aider; nous avons lu les journaux, les périodiques, les livres récents, romans ou livres techniques dans les deux langues afin de trouver des traductions acceptables; nous avons reçu beaucoup de suggestions de la part des usagers du dictionnaire, non seulement d'Angleterre, de France, du Canada et des États-Unis, mais aussi de Belgique, de Suisse, d'Israël, de Suède et même de Roumanie et du Japon (rien de Russie jusqu'à l'heure actuelle !)

Nous avons pris des notes au cours de conversations avec des amis, ou en voyageant. C'est ainsi que nous avons pu inclure parmi bien d'autres : «trous en formation 》, beware of potholes; «pierres errantes» (belgicisme) pour « gravillons » (France), «la gravelle» (Canada), loose chippings, gravel; « aubette» (Belgique), newspaper kiosk; «service du feu» (Suisse) pour «sapeurs-pompiers» (France), fire service, fire station; «benzine», petrol, gas; ou des expressions familières comme «est-ce que je te demande si ta grand-mère fait du vélo?», mind your own bloody business. En ce qui concerne les canadianismes, $\mathbf{M}^{\text {me }}$ Françoise Collin (née Laurendeau), dont le père est avocat ici, à Montréal même, et qui a fait des études à cette université, nous a procuré une liste d'environ 400 mots essentiels. Par exemple :

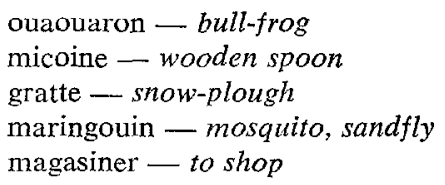

vivoir - living-room poudrerie - blizzard

le grand Montréal - greater Montreal lieutenant-gouverneur - lieutenant governor

Nous avons aussi fait une petite place au franglais, là où il n'y a pas encore de traduction véritablement française. Mais là où les Canadiens ont eu la main heureuse pour créer de véritables mots français pour remplacer le franglais, ils paraissent dans le dictionnaire, par exemple « oléoduc » pour pipeline, ou «ballonpanier $»$ pour basket-ball.

Je voudrais maintenant faire état sous plusieurs rubriques des changements principaux qui sont intervenus pour faire du New Standard le plus moderne et le plus complet des dictionnaires.

1) Aux mots qui existaient déjà en 1934, nous avons ajouté de nouvelles expressions qui n'étaient pas enregistrées alors; je cite parmi des milliers :

agrégatif, -ive, student writing, reading for the agregation

aide, s.m. \& f., aide de l'État, a. aux pauvres, national assistance; aide aux pays en voie de développement, aid for the developing countries

aide (person) m. \& f., aide aux mères, home help; aide familiale, mother's help; s.m. aide auditif, hearing aid aile, aile de mouche, wing nail (of nailed shoes); Aut : prendre un virage sur l'aile, to take a corner on two wheels. Av : l'avion couvre la distance Paris-Dakar d'un coup d'aile, the aircraft makes a non-stop flight from Paris to Dakar

ailé, vis ailée, butterfly screw

albatros, albatros brun, fuligineux, sooty albatross, quaker-bird; albatros hurleur, 
great, wandering albatross ; albatros à sour. cils noirs, black-browed albatross

appareil, dans le plus simple appareil, in the nude; appareil de forage, drilling rig; Tp : gardez l'appareil, hold the line; etc.

bactériologique, bacteriological; guerre bactériologique, germ warfare

bain, Cost : bain de soleil, sun-suit; bain de minuit, bathing in the nude by night

banque, banque de sang, blood bank

barre, Mch : Aut : barre de torsion, torsion bar

base, base de lancement (de fusées), rocketbase; l'anglais de base, basic English

batterie, battery (for faising chicks)

braguette, votre braguette est déboutonnée, you're showing your medals

chambre, chambre à gaz, gas chamber

contrôle des naissances, birth-control, contrôle des changes, exchange control

couverture chauffante, electric blanket

crayon, crayon à bille, ball-point pencil

croiseur, cruiser, c. à propulsion atomique, nucléaire, nuclear-powered cruiser; c. antiaérien, anti-aircraft cruiser; c. de bataille, battle-cruiser ; c. lance-engins, c. lance-missiles, guided-missile cruiser; c. mouilleur de mines, cruiser minelayer; c. porte-hélicoptères, helicopter carrier cruiser; c. protégé, protected cruiser

danse du ventre, belly-dance

danseur, -euse, $\mathrm{Cy}$ : monter une côte en danseuse, to cycle up a hill standing up on one's pedals

débrayer, v., to go on strike, to down tools détergent, detergent, household washing powder

endiguement, Mil : politique d'endiguement, palicy of containment échantillonnage : nous avions 1) Com : making up of samples (of wine), of pattern (of cloth) ; 2) (a) verifying, checking by the samples; (b) gauging by the templet (gauge, gabarit), mais ceci ne permettait pas de traduire : fabriqués à la maison ou à l'atelier les produits représentent un échantillonnage assez êtendu; poteries, tissus, articles de cuir en formaient la plus grosse partie. Nous avons ajouté le sens : range (of goods) et aussi dans un autre sens : choice, selection (of people, etc. for Gallup poll, etc.)

à "passage » nous avons ajouté parmi d'autres sens : il y a du passage ici, (i) there's always a lot of traffic here; (ii) there are always people coming and going, always crowds, here; le passage des harengs, the passage of the herrings, qui précède en raison de la classification, passage de Vénus (sur le disque du soleil), transit of Venus, suivi immédiatement de oiseau de passage, bird of passage, et quelques lignes plus loin, le passage de la libre entreprise au collectivisme, the changeover from free enterprise to collectivism; Radio : T.V. : ce morceau a eu beaucoup de passages, this piece has been broadcast, televised, many times; Civ. E : passage en dessus, p. supérieur, flyover, overpass; passage en dessous, p. inférieur, underpass, etc., et certainement pas comme nous lavait proposé un enthousiaste, fly-under! passage pour piétons, passage clouté, pedestrian crossing; Mil. Av : l'avion effectua deux passages à basse altitude au-dessus des lignes ennemies, the aircraft made two low passes over the enemy lines

soutien-gorge, soutien-gorge pigeonnant, uplift brassiere, etc.

2) Nous avons ajouté comme je l'ai déjà dit plus haut environ 30000 articles représentant tous les sujets, scientifiques, familiers, ou littéraires; si vous le permettez je vais maintenant en citer quelques-uns :

aéroglisseur, hovercraft

aérogramme, aerogram, air-letter

aéroporté, airborne

aérosol, aerosol

aérotransporter, to carry by air

afro-asiatique, Afro-Asian

agrafeuse, stapler

agriflamme, flame gun

aide-ouie, hearing aid

alaire, charge alaire, wing load, surface alaire, wing area appareil, appareil à cousin d'air, hovercraft appontage, landing on flight-deck of aircraft carrier; officier d'appontage, landing officer

autoroute, motorway, U.S. : speedway

auto-stoppeur, hitch-hiker

balconet, half-cup brassiere

bathyscaphe, bathyscaph

bébé-éprouvette, test-tube baby

benzédrine, benzedrine

bidonville, shanty town 
chèque-repas, luncheon voucher, meal token craquage, Petr. Ind. : cracking (of heavy oil,) suivi de six lignes d'exemples et de traductions techniques

crayon-lèvres, lipstick

crêperie, pancake bar déshabillage, strip-tease dessous-de-table, golden handshake; verser un dessous-de-table, to pay (s.o. a bribe) ; (in property transactions) to pay a sum over and above the stated price, under the counter

déstaliniser, to destalinize

déstalinisation, destalinization

Je pourrais continuer cette liste; il y a trente mille nouveaux mots comme je l'ai dit plus haut. Déjà maintenant nous commençons à faire la liste des nouveaux mots qui ont paru depuis quatre ans. En effet il nous a fallu donner le bon à tirer pour la lettre $\mathrm{C}$ en 1968. Ceci signifie que nous n'avons pas pu inclure « crayonfeutre », felt pen, dont l'usage ne s'est répandu que depuis cette date. Mais avant de publier un supplément à la partie français-anglais, nous devons terminer la partie anglais-français. Et nous estimons que ce travail va nous prendre au bas mot encore cinq ou six ans.

La publication de la partie français-anglais du Harrap New Standard Dictionary aurait pris beaucoup plus de temps encore si ma femme Margaret n'avait pas mis la main à la pâte au point qu'avec l'aide de sa sœur Muriel, elle a préparé à peu près les trois quarts du manuscrit. Nous avons eu l'aide également d'un certain nombre de collaborateurs au bureau qui ont entrepris un travail prodigieux de recherche. Parmi eux je voudrais mentionner $M^{\text {lies }}$ Vivien Flynn et Christine Fontan, $\mathrm{M}^{\text {mes }}$ Elizabeth Anne Strick et Valerie Deane (qui m'a quitté pour se marier et venir vivre au Canada), et en dehors du bureau, des dizaines de personnes. Les épreuves ont été lues par moi-même depuis $A$ jusqu'à $Z$ et par quatre lecteurs qui ont attiré notre attention sur un certain nombre d'omissions ou d'erreurs.

Le New Standard French and English Dictionary donne aussi la notation phonétique de chaque mot. Ceci signifie que nous avons au moins 100000 notations phonétiques, ce qui est beaucoup plus que nous trouvons dans les dictionnaires spécialisés. M. Quemada nous a apporté une aide considérable dans cette tâche.

Je voudrais également remercier les imprimeurs, MM. Pitman de Bath pour l'excellent travail qu'ils ont fait, depuis la composition jusqu'à l'impression des soixante-quinze tonnes de papier qu'il a fallu utiliser pour cette première impression. Leur travail n'a pas toujours été facile, car une partie du manuscrit était écrite à la main (et non pas dactylographiée pour éviter des erreurs de frappe ce qui aurait nécessité une double lecture). Ceci a eu pour résultat de causer des coquilles amusantes que nous avons corrigées à la lecture des épreuves, et dont je cite maintenant quelques-unes : "urine chargée », clouded wine (pour urine); «binoclard», person who wears gloves (pour glasses); «chien dressé à la propreté », horse-trained dog (pour house-trained dog) ; «l'industrie de la chaussure », the boat and shoe industry (pour boot and shoe industry) ; «phantasme», official illusion (pour optical illusion). Nous espérons n'avoir rien laissé d'autre. Ces coquilles, je me hâte de le dire, doivent être imputées à notre écriture et non pas au compositeur qui pouvait s'attendre à tout dans un ouvrage qui contient des choses étranges comme le terme d'informatique «multiplet», eight-bit byte. 